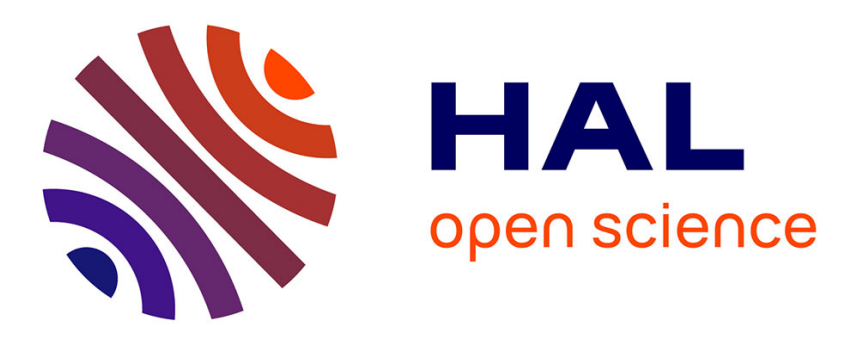

\title{
Analyzing sound speed fluctuations in shallow water from group-velocity versus phase-velocity data representation
}

Philippe Roux, William A. Kuperman, Bruce D Cornuelle, Florian Aulanier, William S. Hodgkiss, Hee Chun Song

\section{To cite this version:}

Philippe Roux, William A. Kuperman, Bruce D Cornuelle, Florian Aulanier, William S. Hodgkiss, et al.. Analyzing sound speed fluctuations in shallow water from group-velocity versus phase-velocity data representation. Journal of the Acoustical Society of America, 2013, 133 (4), pp.1945-1952. 10.1121/1.4792354 . hal-00841130

\author{
HAL Id: hal-00841130 \\ https://hal.science/hal-00841130
}

Submitted on 5 Nov 2013

HAL is a multi-disciplinary open access archive for the deposit and dissemination of scientific research documents, whether they are published or not. The documents may come from teaching and research institutions in France or abroad, or from public or private research centers.
L'archive ouverte pluridisciplinaire HAL, est destinée au dépôt et à la diffusion de documents scientifiques de niveau recherche, publiés ou non, émanant des établissements d'enseignement et de recherche français ou étrangers, des laboratoires publics ou privés. 


\title{
Analyzing sound speed fluctuations in shallow water from group-velocity versus phase-velocity data representation
}

\author{
Philippe Roux ${ }^{\text {a) }}$ \\ Institut des Sciences de la Terre, Université Joseph Fourier \& Centre National de la Recherche Scientifique \\ (CNRS), Grenoble, France
}

W. A. Kuperman and Bruce D. Cornuelle
Scripps Institution of Oceanography, UC San Diego, La Jolla, California 93027

Florian Aulanier

Laboratoire Grenoble Images Parole Signal Automatique, Institut Polytechnique de Grenoble \& Centre National de la Recherche Scientifique (CNRS), Grenoble, France

\author{
W. S. Hodgkiss and Hee Chun Song \\ Scripps Institution of Oceanography, UC San Diego, La Jolla, California 93027
}

(Received 25 June 2012; revised 14 January 2013; accepted 30 January 2013)

\begin{abstract}
Data collected over more than eight consecutive hours between two source-receiver arrays in a shallow water environment are analyzed through the physics of the waveguide invariant. In particular, the use of vertical arrays on both the source and receiver sides provides source and receiver angles in addition to travel-times associated with a set of eigenray paths in the waveguide. From the travel-times and the source-receiver angles, the eigenrays are projected into a group-velocity versus phase-velocity ( $\mathrm{Vg}-\mathrm{Vp}$ ) plot for each acquisition. The time evolution of the Vg-Vp representation over the 8.5-h long experiment is discussed. Group speed fluctuations observed for a set of eigenrays with turning points at different depths in the water column are compared to the BruntVäisälä frequency. (C) 2013 Acoustical Society of America. [http://dx.doi.org/10.1121/1.4792354]
\end{abstract}

PACS number(s): 43.30.Cq, 43.30.Re, 43.60.Fg [MS]

Pages: $1945-1952$

\section{INTRODUCTION}

In shallow-water oceans, multipath propagation caused by reflection and/or refraction of acoustic eigenrays usually results in a complex interference pattern at the receiver. It turns out that the interference pattern at a slightly shifted frequency keeps the same structure, which is a robust feature of the ocean propagation that is described by a scalar parameter referred to as the waveguide invariant (Chuprov, 1982; Brekhovskikh and Lysanov, 2003; Jensen et al., 2011; D’Spain and Kuperman, 1999). The waveguide invariant is also descriptive of the shift in the interference pattern associated with changes in range or environmental parameters (Grachev, 1993; Kim et al., 2003; Quijano et al., 2010). This physics can be interpreted as a strong relationship between the change in group-speed with respect to the change in phase-speed for a set of eigenrays (or eigenmodes) that remains valid even in a waveguide that shows environmental fluctuations. As this robustness suggests model-independent processing, an assortment of applications have been recently developed, including range localization (Thode, 2000; Cockrell and Schmidt, 2010; Fupo and Yue, 2011), time-reversal focusing (Song et al., 1998; Kim et al., 2003) and reverberation and active sonar processing (Yang, 2003; Hailing and Krolik, 2007; Rouseff and Zurk, 2011).

Previous studies on the frequency dependence of interference patterns in fluctuating environments (Badiey et al., 2007) made the connection between the acoustic perturba-

\footnotetext{
a) Author to whom correspondence should be addressed. Electronic mail: philippe.roux@obs.ujf-grenoble.fr
}

tions and a specific ocean environment. In the present study, the concept of the waveguide invariant is applied to a fluctuating shallow-water ocean environment through a large set of eigenrays that are extracted from the data recorded between two source-receiver arrays. The time evolution of the group-velocity versus phase-velocity $(\mathrm{Vg}-\mathrm{Vp})$ data representation can provide quantitative information regarding the sound speed fluctuations at the thermocline that compare favorably with independent sound speed measurements in the water column, although some discrepancies remain. In particular, the $\mathrm{Vg}-\mathrm{Vp}$ data analysis shows that the physical origin of the largest fluctuations is closely identified with abrupt changes in the waveguide invariant.

This paper is structured as follows. Section II deals with a description of the lexperimental setup and data acquisition at sea. The beamforming process that is performed on the source-receiver arrays that transforms the waveguide transfer matrix into a set of eigenrays is then presented. In Sec. III, the eigenray travel-times and incident angles are projected into the $\mathrm{Vg}-\mathrm{Vp}$ representation, from which the waveguide invariant is calculated for reflected and refracted ray paths. The time evolution of the $\mathrm{Vg}-\mathrm{Vp}$ plot along the 8.5-h long repetitive acquisition is discussed. Quantitative measurements of the group velocity changes are compared with the sound speed measurements in the water column.

\section{EXPERIMENTAL CONFIGURATION AND DATA PROCESSING}

The FAF05 (Focused Acoustic Field 2005) experiment was conducted in July 2005, to the north of Elba Island, Italy, 
with repetitive acoustic acquisitions between two sourcereceiver vertical arrays that were separated by a distance $R=4.071 \mathrm{~km}$ in a $123 \mathrm{-m}$ deep waveguide [Fig. 1(a)]. The source (SA) and receiver (RA) arrays are made up of, respectively, 29 equally spaced transducers that span $78 \mathrm{~m}$, and 32 equally spaced hydrophones that cover $62 \mathrm{~m}$ of the water column (Roux et al., 2004). The time-domain pressure field $p\left(t, z_{r}, z_{s}\right)$ transmitted from a transducer at depth $z_{s}$ is recorded on each receiver of the vertical hydrophone array at depth $z_{r}$. The transducers have a central frequency $F_{0}=3.2 \mathrm{kHz}$ with a $\Delta \omega=1 \mathrm{kHz}$ bandwidth. The source signals were $200-\mathrm{ms}$ linear frequency modulated chirps that were compressed after reception to their pulse equivalent by cross-correlation. This provided $40 \mathrm{~dB}$ of signal-to-noise ratio for broadband reception with power-limited transmission. The source signal was transmitted sequentially from each transducer, with a separation between transmissions of $250 \mathrm{~ms}$ greater than the channel dispersion time. On Julian Day 197, the acquisition of the waveguide transfer function was completed in $7 \mathrm{~s}$ and repeated every $20 \mathrm{~s}$, for a total duration of $8.5 \mathrm{~h}$ of recordings that were performed to monitor fluctuations of the oceanic waveguide.

During the course of the experiment, a collection of conductivity-temperature-depth (CTD) casts were taken at multiple locations close to the arrays. The average and standard deviation of the depth-dependent sound speed profiles measured by the CTD casts is shown in Fig. 1(b). The sound speed profile is downward refracting, with strong gradients at thermoclines $\mathrm{A}$ and $\mathrm{B}$, at depths of 35 and $15 \mathrm{~m}$, respectively. Internal wave displacements between 15 and $35 \mathrm{~m}$ are expected to be the dominant source of sound speed variability on minute-to-hour time scales, which produce the greatest changes at the thermocline depths.

The acoustic data were extensively described in Roux et al. (2008). The depth-time representation of the pressure field received on the RA clearly displayed an interference pattern caused by the superposition of several acoustic wavefronts refracted and/or reflected by the waveguide interfaces [see Fig. 3(a) in Roux et al., 2008]. The sound speed variability at the thermocline also produced significant changes in the waveguide transfer function over time (see Fig. 5 in Roux et al., 2008).

In Roux et al. (2008), the data analysis was performed through a double beamforming (DBF) algorithm that was simultaneously applied to the SA and RA on each acquisition of the waveguide transfer matrix. When applied to broadband data, DBF provides the identification of echo arrivals by their launch and receive angles $\left(\theta_{r}, \theta_{s}\right)$ and traveltimes (Iturbe et al., 2009a; Marandet et al., 2011; Sarkar et al., 2012). The DBF processing can be summarized as the projection of the acoustic data from the physical domain ( $t$, $\left.z_{r}, z_{s}\right)$ to the time-angle domain $\left(t, \theta_{r}, \theta_{s}\right)$ through the mathematical transformation

$$
\begin{aligned}
\tilde{p}\left(t, \theta_{r}, \theta_{s}\right)= & \frac{1}{N_{r} N_{s}} \sum_{i=1}^{N_{r}} \sum_{j=1}^{N_{s}} p\left(t+\tau\left(\theta_{r}, z_{i}\right)\right. \\
& \left.+\tau\left(\theta_{s}, z_{j}\right), z_{i}, z_{j}\right)
\end{aligned}
$$

where $N_{r}$ and $N_{s}$ are the number of receivers and transducers on the SAs and RAs. To limit the angle uncertainties caused by beamforming in depth-dependent and time-varying sound speed profiles, the DBF algorithm was restricted to sourcereceiver subarrays made of 9 consecutive transducers on the SA and 11 consecutive hydrophones on the RA, with their upper elements always below a depth of $70 \mathrm{~m}$. At this depth, plane-wave beamforming can be applied with a constant sound speed $c=1507.5 \mathrm{~m} / \mathrm{s}$, which means that the timedelays in Eq. (1) are calculated as $\tau(\theta, z)=\left(z-z_{0}\right) \sin \theta / c$ for a subarray centered at depth $z_{0}$.

For any pair of source-receiver subarrays chosen among the 17 bottom transducers and the 19 bottom hydrophones of the SA and RA, respectively [Fig. 1(b)], a set of 12 beams
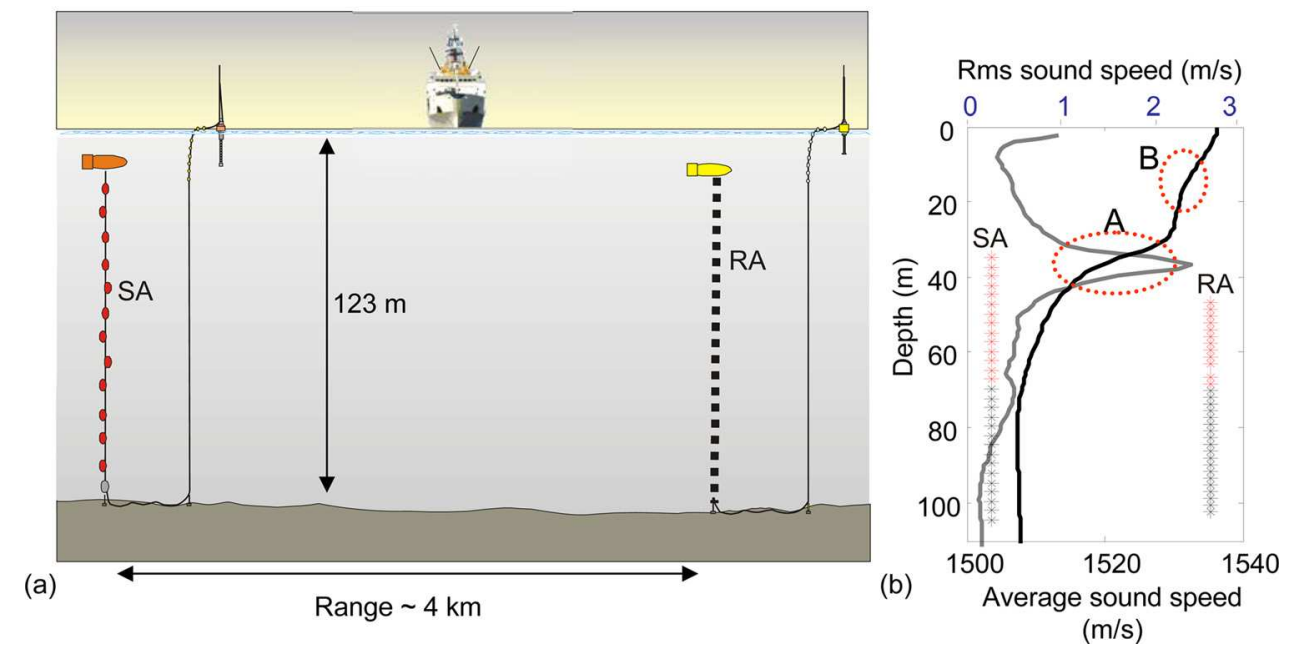

FIG. 1. (Color online) Schematic of the experimental setup at sea. (a) On the left, a bottom-moored SA that consists of 29 transducers centered at $3.5 \mathrm{kHz}$ that cover $78 \mathrm{~m}$ of the water column. On the right is a bottom-moored RA that consists of 32 hydrophones with a 90 -m aperture. The two surface buoys contain batteries and the radio-frequency telemetry hardware for data communication with a ship. The water depth is $123 \mathrm{~m}$ and the distance between the SA and the RA is $4.071 \mathrm{~km}$. (b) Mean (black) and rms (gray) sound speed profiles for the region. The rms sound speed axis is at the top and the mean sound speed axis is at the bottom. The source and receiver depths are indicated by the plus signs to the left and right of the figure, respectively. The black symbols correspond to the 19 bottom hydrophones and the 17 bottom transducers of the RA and SA, respectively. The dashed circles show the locations of two thermoclines in the water depth, as thermoclines $\mathrm{A}$ and $\mathrm{B}$, at depths of $\sim 35$ and $\sim 15 \mathrm{~m}$, respectively. 
could be isolated with source-receiver angles up to $\sim 15^{\circ}$ and angle resolution less than $1^{\circ}$ (see Fig. 10 in Roux et al., 2008). After DBF, each beam detected in the $\left(t, \theta_{r}, \theta_{s}\right)$ domain is matched to its ray path equivalent that connects the subarray centers through the closest arrival time and launch/ receiver angles. In the following, such beams are equivalently called eigenrays. Finally, a total of $\sim 1000$ beams were extracted and identified as eigenrays from among $9 \times 9=81$ source-receiver subarray configurations for one single acquisition.

Using this complete set of eigenrays, the array tilts can first be measured and corrected separately at the SA and RA. Indeed, as the sound speed is assumed to be constant over the bottom part of the arrays where DBF is performed, the launch and receive angles, $\theta_{s}$ and $\theta_{r}$ respectively, should be equal or opposite. In practice, however, the angle uncertainties through $\mathrm{DBF}$, the sound speed heterogeneities, and the array tilt can affect the estimated values $\tilde{\theta}_{s}$ and $\tilde{\theta}_{r}$, such that

$$
\begin{aligned}
& \tilde{\theta}_{s}=\theta_{s}+n_{s}+\Delta \theta_{s}, \\
& \tilde{\theta}_{r}=\theta_{r}+n_{r}+\Delta \theta_{r},
\end{aligned}
$$

where $n_{s}$ (respectively, $n_{r}$ ) and $\Delta \theta_{s}$ (respectively, $\Delta \theta_{r}$ ) account for the random noise and the array tilt on the SA and RA, respectively. Despite the noise on the DBF angle estimates, the distributions of $\tilde{\theta}_{s}+\tilde{\theta}_{r}$ and $\tilde{\theta}_{s}-\tilde{\theta}_{r}$ calculated for the complete set of eigenrays reveal two peaks around zero that are associated with $\Delta \theta_{s}+\Delta \theta_{r}$ and $\Delta \theta_{s}-\Delta \theta_{r}$, from which $\Delta \theta_{s}$ and $\Delta \theta_{r}$ can be measured separately.

The physical interpretation of $\Delta \theta_{s}$ and $\Delta \theta_{r}$ is two-fold. First, the range and depth-dependent sound speed heterogeneities in the water column (mostly at the thermocline) induce travel-time changes as well as launch and receive angle fluctuations for each eigenray (Roux et al., 2008). The angle fluctuations depend on the eigenray path with respect to the sound speed heterogeneities (Aulanier et al., 2011). When the source-receiver angle fluctuations are averaged over 1000 eigenrays that span the whole waveguide, the contribution associated with sound speed heterogeneities averages out. On the other hand, the array tilt remains the same for every eigenray angle measurement, and it should be the final dominant contribution when taking into account the whole set of 1000 extracted eigenrays. Note, however, that only the projection of the array tilts on the SA-RA plane is effectively extracted from the DBF measurements.

The time evolution of $\Delta \theta_{s}$ and $\Delta \theta_{r}$ over the $8.5 \mathrm{~h}$ of the experiment is presented in Fig. 2. As expected for moored vertical arrays in a quiet environment (Hodgkiss et al., 1995), the array tilts at depths are small [always lower than $0.5^{\circ}$, Fig. 2(b)]. They can be compared to water currents evolution measured at a depth of $90 \mathrm{~m}$ from two upward-looking Acoustic Doppler Current Profilers (ADCPs) mounted on 2 separate moorings $1.7 \mathrm{~km}$ apart in the vicinity of the acoustic arrays [Fig. 2(a)]. The current evolution shows an average period of $17 \mathrm{~h}$ with a noticeable phase shift for JD197 between the two locations. The current periodicity compares favorably with the array tilt evolution. The tilts appear to evolve independently on the 2 arrays that are $4 \mathrm{~km}$ apart.
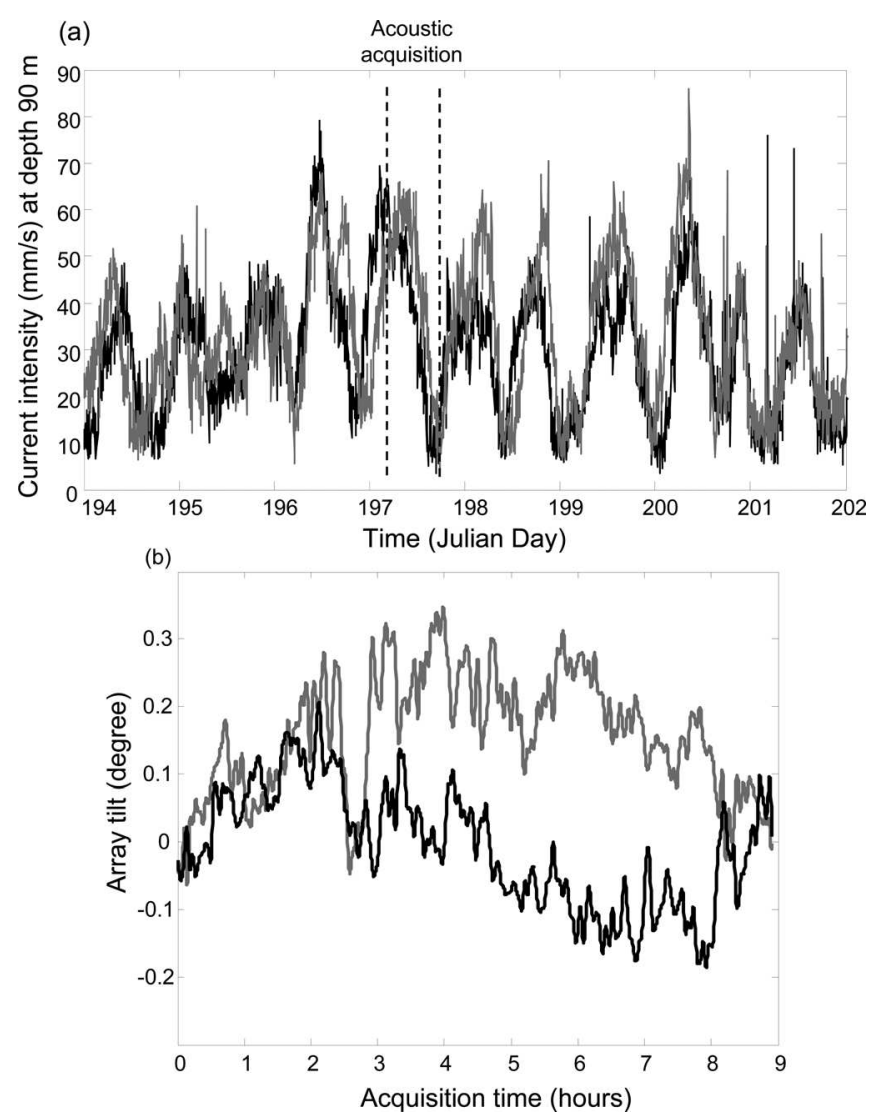

FIG. 2. (a) Time evolution of the currents' intensity at 90 -m depth during 8 consecutive days. The currents were measured from two ADCPs mounted on moorings at $1.7 \mathrm{~km}$ of each other (gray and black). The dashed vertical lines show the interval time of the acoustic acquisition on JD 197. (b) Time evolution of the array tilts ( $\Delta \theta_{s}$ in gray for the SA, and $\Delta \theta_{r}$ in black for the RA) as measured from the acoustic data.

In the following, the time-evolving array tilt inferred from the total dataset is used to correct for the launch and receive angles extracted from the $\mathrm{DBF}$ algorithm for each eigenray.

\section{VG VERSUS VP REPRESENTATION}

For each eigenray characterized by $\left(t_{0}, \theta_{r}^{0}, \theta_{s}^{0}\right)$, the group velocity is calculated as the range $R$ divided by the traveltime

$$
V_{g}^{0}=\frac{R}{t_{0}} .
$$

The phase velocity is defined in this paper as the average ratio of the local sound speed at the source-receiver arrays $\left(c_{s}=c_{r}=c=1507.5 \mathrm{~m} / \mathrm{s}\right)$ to the cosine of the incident grazing angle on the array

$$
V_{p}^{0}=\frac{1}{2}\left(\frac{c_{s}}{\cos \left(\theta_{s}^{0}\right)}+\frac{c_{r}}{\cos \left(\theta_{r}^{0}\right)}\right) .
$$

Figure 3 represents the DBF data plotted as the $\mathrm{Vg}-\mathrm{Vp}$ for one acquisition of the waveguide transfer matrix. The data and theoretical Vg-Vp plots, which were obtained from tracing rays through the time-averaged sound speed profile shown 


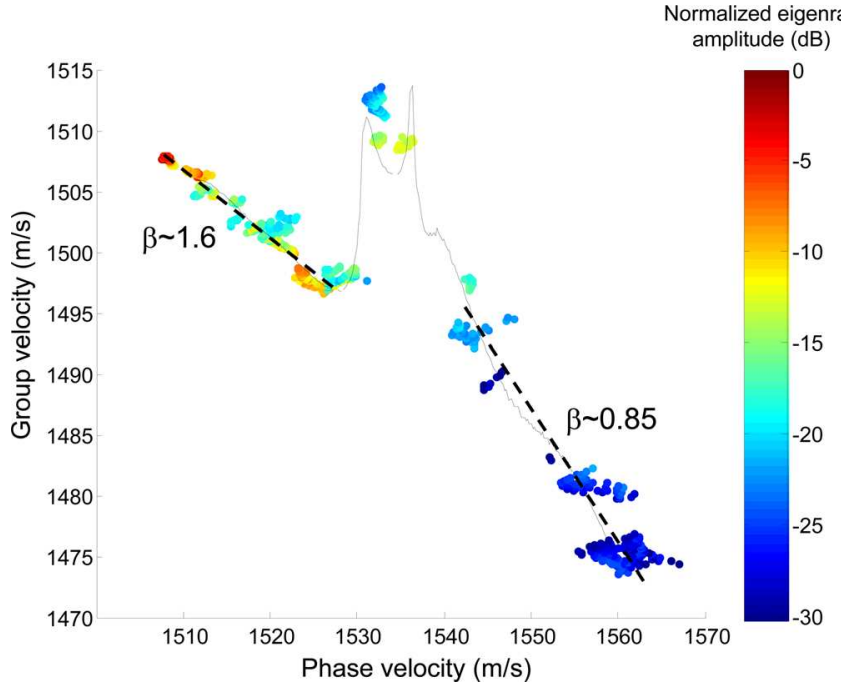

FIG. 3. (Color online) Vg-Vp representation of the acoustic data for one acquisition of the point-to-point response between every source and every receiver of the source-receiver arrays. Each spot corresponds to one of the $\sim 1000$ beams extracted through DBF. The normalized beam amplitude is plotted in $\mathrm{dB}$. The black curve is the ray-tracing computation obtained from the average sound speed profile. The waveguide invariant $\beta$ was calculated (dashed lines) for the beams with $\mathrm{Vp}<1528 \mathrm{~m} / \mathrm{s}$ and $\mathrm{Vp}>1540 \mathrm{~m} / \mathrm{s}$, respectively.

in Fig. 1(b), compare well with one another. The gaps observed in the data are due to the limited array apertures that prevent a continuous sampling in angle or phase velocity.

The waveguide invariant $\beta$ is defined from a functional relationship between the group and phase velocities (or, equivalently, the group and phase slowness). We can then express individual group and phase velocities as a Taylor expansion around the average group and phase velocities of a set of eigenrays. From Jensen et al. (2011), it can be shown that

$$
\frac{1}{\beta}=-\frac{d\left(1 / V_{g}\right)}{d\left(1 / V_{p}\right)}=-\left(\frac{V_{p}}{V_{g}}\right)^{2} \frac{d V_{g}}{d V_{p}},
$$

so that a $\mathrm{Vg}-\mathrm{Vp}$ plot provides a direct measurement of the waveguide invariant. From Fig. 3, the eigenrays can be sorted into two groups. The eigenrays are either refracted at thermoclines A and/or B for $\mathrm{Vp}<1538 \mathrm{~m} / \mathrm{s}$ (the average sound speed at the surface), or they are reflected at the airwater interface for $\mathrm{Vp}>1538 \mathrm{~m} / \mathrm{s}$. Note that (1) both sets of eigenrays interact with the bottom (Fig. 4); and (2) the surface-reflected eigenrays have a lower amplitude than the refracted eigenrays due to reflection loss.

The surface-reflected eigenrays are characterized by a waveguide invariant $\beta \sim 0.85$, which is in agreement with the Pekeris waveguide approximation $\beta=\cos ^{2} \theta$ where $\theta$ is the critical angle that depends on the bottom properties (Jensen et al., 2011; Shang et al., 2012). For the refractedreflected eigenrays that are typical in downward refracting profiles [see eigenrays (2), (3), and (4) in Fig. 4], the waveguide invariant $\beta>1$ strongly depends on the sound speed change at the thermocline. The value $\beta \sim 1.6$ is in agreement with values found in Jensen et al. (2011). The increased value for $\beta$ for the refracted eigenrays occurs because the propagation is dominated by the low-order acoustic modes bound from above by the thermocline (at a depth of $35 \mathrm{~m}$ ), with no interaction with the sea surface (Rouseff and Leigh, 2002; Rouseff and Spindel, 2002). Note that negative values of the waveguide invariant $(\beta \sim-3)$ are also computed for purely refracted eigenrays in the deep-water ocean (Kuperman et al., 2002; Harrison, 2011).

The same processing was performed over $8.5 \mathrm{~h}$ of repetitive recordings to produce the average $\mathrm{Vg}-\mathrm{Vp}$ representation. In this case, a $\mathrm{Vg}-\mathrm{Vp}$ grid was defined with grid intervals $\delta V_{g}=1 \mathrm{~m} / \mathrm{s}$ and $\delta V_{p}=1 \mathrm{~m} / \mathrm{s}$. These grid intervals, respectively, correspond to a small change in travel-time $\delta t$ $\sim 2 / \Delta \omega=2 \mathrm{~ms}$ or incident angle $\delta \theta \sim 0.3^{\circ}$, according to Eqs. (3) and (4). For the 8.5 -h long acoustic recordings, the eigenray amplitudes that fall into the same cell $\left(\delta V_{g}, \delta V_{p}\right)$ are then accumulated, to produce the average $\mathrm{Vg}-\mathrm{Vp}$ plot and corresponding standard deviation (Fig. 5). The phase velocity scale is also shown as the absolute eigenray angle at the source and receiver.

As expected, the surface-reflected eigenrays [Vp $>1540 \mathrm{~m} / \mathrm{s}$, see eigenray (5) in Figs. 4 and 5(a)] show a large dispersion in the $\mathrm{Vg}-\mathrm{Vp}$ representation and strong amplitude fluctuations, that is consistent with the impact of the rough sea surface on the eigenray travel-time, launchreceiver angles, and amplitude (Roux et al., 2010). On the other hand, the beams that are refracted far below the main thermocline [ $\mathrm{Vp} \sim 1510 \mathrm{~m} / \mathrm{s}$, see eigenray (1) in Figs. 4 and $5(\mathrm{a})]$ are very focused in the $\mathrm{Vg}-\mathrm{Vp}$ representation, which means that both their arrival time, launch, and receiver angles remain stable over repetitive recordings. Finally, the most interesting eigenrays are those that are refracted at thermocline $\mathrm{A}$, or between thermocline $\mathrm{A}$ and $\mathrm{B}(\mathrm{Vp} \sim 1525$ to $1535 \mathrm{~m} / \mathrm{s}$ ) since their travel-time, launch-receiver angles, and amplitude fluctuations are strongly related to the acoustic interaction with internal waves.

To go one step further, Fig. 6 shows the time evolution of the $\mathrm{Vg}-\mathrm{Vp}$ data with no eigenray amplitude information but with the group velocity shown as colors. This study was limited to $\mathrm{Vp}<1545 \mathrm{~m} / \mathrm{s}$ where acoustic fluctuations are not caused by the rough air-water interface but mostly come

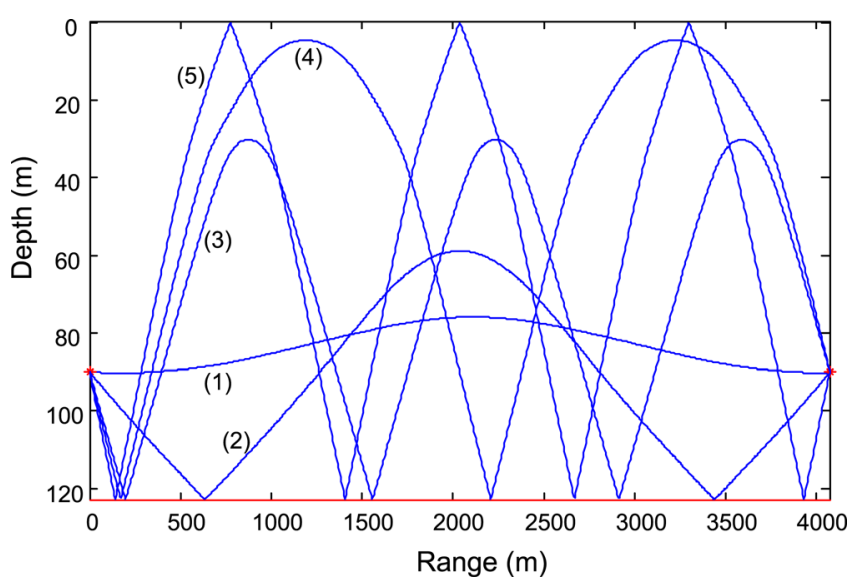

FIG. 4. (Color online) Set of eigenrays propagating between two elements of the SA and RA (at a depth of $90 \mathrm{~m}$ ) with different phase and group velocities. (1) $\mathrm{Vp}=1508 \mathrm{~m} / \mathrm{s}, \mathrm{Vg}=1508 \mathrm{~m} / \mathrm{s}$; (2) $\mathrm{Vp}=1510 \mathrm{~m} / \mathrm{s}, \mathrm{Vg}=1506 \mathrm{~m} / \mathrm{s}$; (3) $\mathrm{Vp}=1526 \mathrm{~m} / \mathrm{s}, V \mathrm{~g}=1497 \mathrm{~m} / \mathrm{s} ;$ (4) $\mathrm{Vp}=1536 \mathrm{~m} / \mathrm{s}, V g=1511 \mathrm{~m} / \mathrm{s}$; (5) $\mathrm{Vp}=1548 \mathrm{~m} / \mathrm{s}, \mathrm{Vg}=1487 \mathrm{~m} / \mathrm{s}$. 


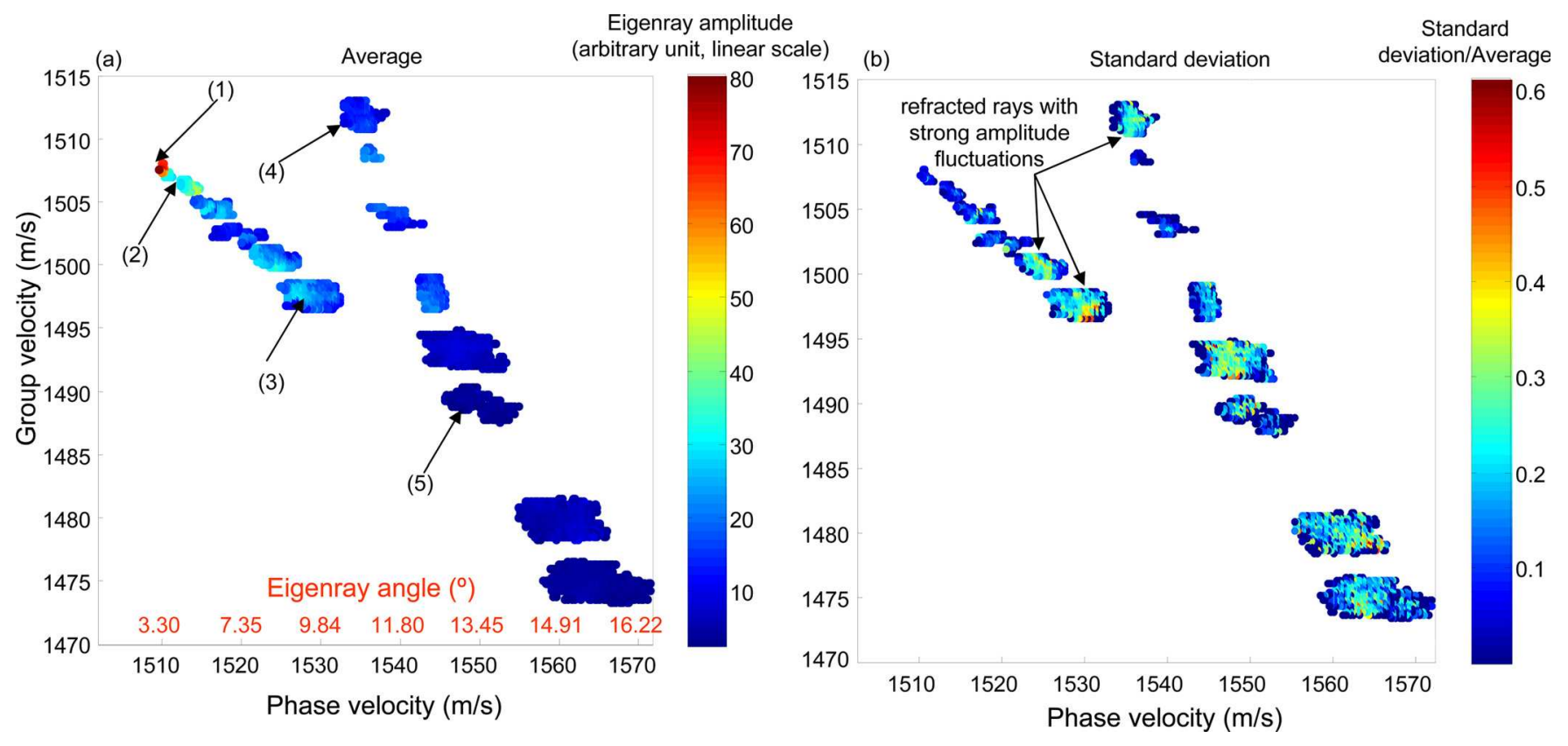

FIG. 5. (Color online) Average (a) and standard deviation (b) of the Vg-Vp data accumulated over the $8.5 \mathrm{~h}$ of the acoustic acquisition. (a) The beam amplitude is plotted as a linear color bar. The arrows pointing at numbers (1) to (5) refer to the eigenrays displayed in Fig. 4. Both the phase velocity and eigenray launch/receiver angles are indicated for the $x$-axis. (b) The color bar corresponds to the standard deviations of the beam amplitude normalized by the beam average amplitude. The arrows indicate the refracted eigenrays at thermoclines A and B with strong amplitude fluctuations.

from the interaction with sound speed perturbations at the thermoclines.

When the $\mathrm{Vg}-\mathrm{Vp}$ average is subtracted from this data representation, the time evolution of the group velocity fluctuations becomes more visible in Fig. 7. The effects of internal wave interactions with the acoustic field are observed at thermocline A (for $\mathrm{Vp} \sim 1523 \mathrm{~m} / \mathrm{s}$ ), and even more so at thermocline B (where $\mathrm{Vp} \sim 1531 \mathrm{~m} / \mathrm{s}$ ). Fluctuations are also clearly observed for $\mathrm{Vp} \sim 1537 \mathrm{~m} / \mathrm{s}$, which correspond to rays that either reflect on the surface or refract just before the air-water interface (Kuperman et al., 2001). However, the lack of data in Fig. 7 at these phase velocities does not allow for a clear interpretation.

Group velocity perturbations of $\pm 8 \mathrm{~m} / \mathrm{s}$ are in agreement with the root-mean-square (rms) sound speed in Fig. 1(b) $(\sim$ slightly above $2 \mathrm{~m} / \mathrm{s})$. For three different phase velocities $(\mathrm{Vp}=1512 \mathrm{~m} / \mathrm{s}$, nearly no fluctuation, $\mathrm{Vp}=1523 \mathrm{~m} / \mathrm{s}$ at thermocline $A, V p=1531 \mathrm{~m} / \mathrm{s}$ at thermocline B), Fig. 8 shows the corresponding group velocity fluctuations, $\Delta \mathrm{Vg}$, along the 8.5 -h recording time. No clear periodicity is observed at thermoclines A and B but rather some burst of group velocity fluctuations on time-scales ranging from 10 to $20 \mathrm{~min}$ which is compatible with the presence of solitary internal waves (Badiey et al., 2007). Note that such $\Delta \mathrm{Vg}$ does not look correlated with the array tilt (Fig. 2), which confirms that the array tilts and the internal wave interactions can be separated from the acoustic data.

\section{CONSISTENCY OF DATA ANALYSIS WITH DIFFRACTION PHYSICS THROUGH THE SENSITIVITY KERNEL APPROACH}

Figure 9 shows the average time autocorrelation function of the group velocity fluctuations obtained from Fig. 8 at three different phase velocities. As expected from Fig. 7, the shortest decorrelation time ( $\sim$ a few minutes) is obtained for eigenrays with a turning point at thermocline B (depth $\sim 15 \mathrm{~m}$ and $\mathrm{Vp}=1531 \mathrm{~m} / \mathrm{s}$ ).

As these variations are assumed to be due to internal waves, the Brunt-Väisälä (BV) frequency was computed from the CTD measurements, as $N^{2}(z)=-g / \rho(\partial \rho / \partial z)$ where $g$ is the gravitational acceleration and $\rho(z)$ is the local density in the fluid that depends on both temperature and salinity. In oceanography, BV measures the frequency of the restoring force that controls the oscillation of a fluid particle in a stable and stratified ocean. The BV frequency versus

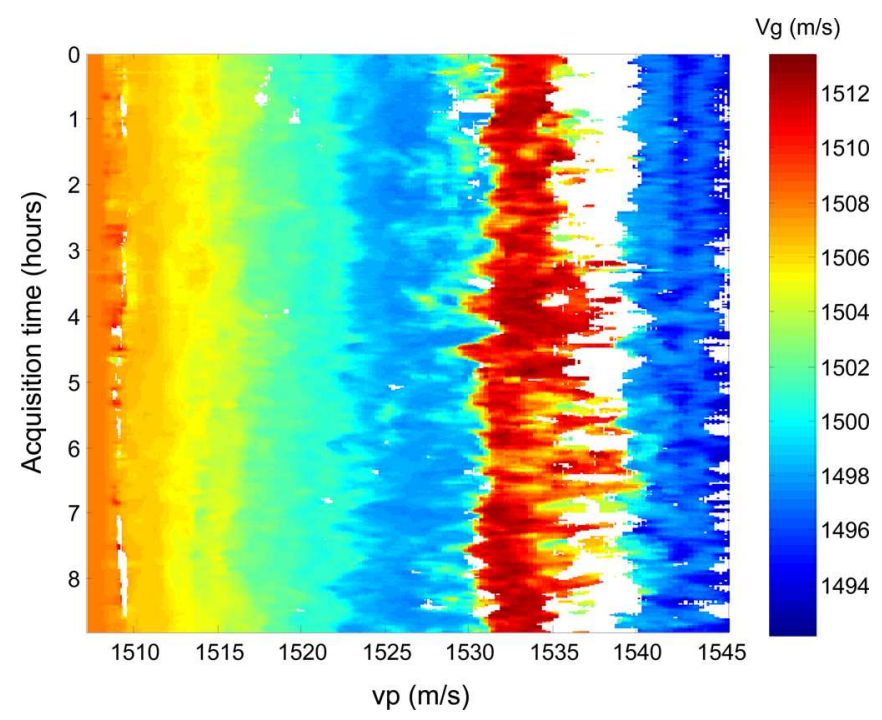

FIG. 6. (Color online) Time evolution of the Vg-Vp acoustic data along the $8.5 \mathrm{~h}$ of acoustic acquisition. The group velocity is plotted as a linear color bar. The white areas correspond to places with missing $\mathrm{Vg}-\mathrm{Vp}$ data. 


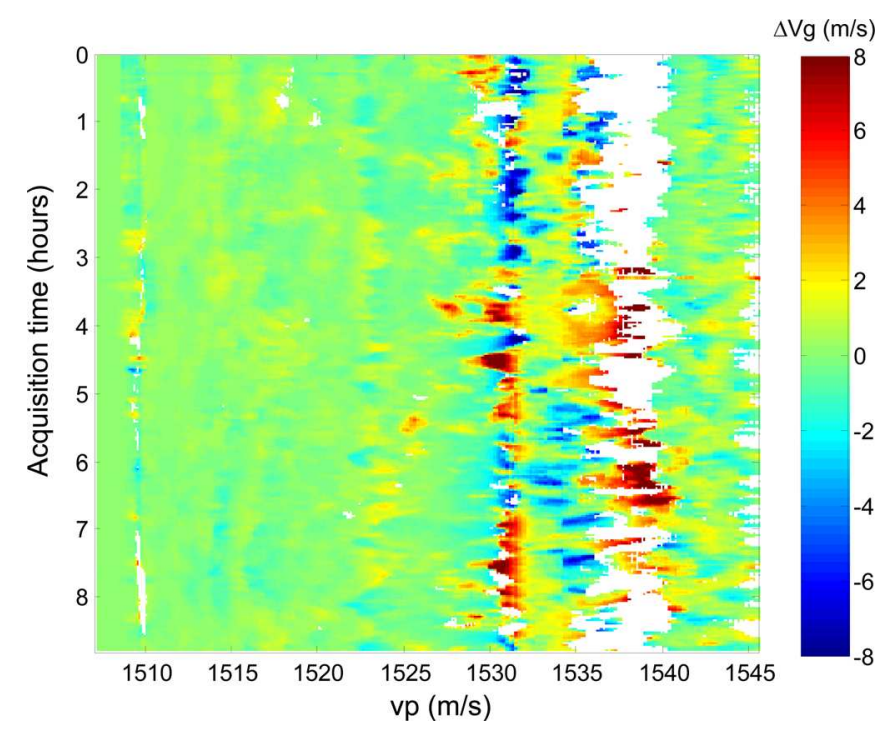

FIG. 7. (Color online) Time evolution of the group velocity fluctuations $\Delta \mathrm{Vg}$ along the $8.5 \mathrm{~h}$ of acoustic acquisition. The dark areas indicate places with no Vg-Vp acoustic data. The white areas correspond to places with missing Vg-Vp data.

depth relates to internal wave propagation and provides a useful description of oceanic stability.

As expected, the dominant $\mathrm{BV}$ frequency, $F_{\mathrm{BV}}$, is at thermocline A, which is the place of the highest sound speed gradients in the water column [Fig. 10(a)]. The value $F_{\mathrm{BV}}$ $\sim 20$ cycles/h is in agreement with the shortest coherence time of the acoustic measurements. However, Fig. 10(a) shows that the BV frequency should be maximum at thermocline A for $\mathrm{Vp} \sim 1523 \mathrm{~m} / \mathrm{s}$ (for a turning point at depth $\sim 35 \mathrm{~m}$ ) when the acoustic data in Fig. 9 have the shortest coherence time at thermocline $\mathrm{B}$, for $\mathrm{Vp} \sim 1531 \mathrm{~m} / \mathrm{s}$ (for a turning point at depth $\sim 15 \mathrm{~m}$ ). Such discrepancy cannot be explained by the up and down motion of the thermocline as the internal wave passes since these two turning point depths are too far from each other.

We suggest instead an explanation based on diffraction physics that requires the computation of the travel-time sen-

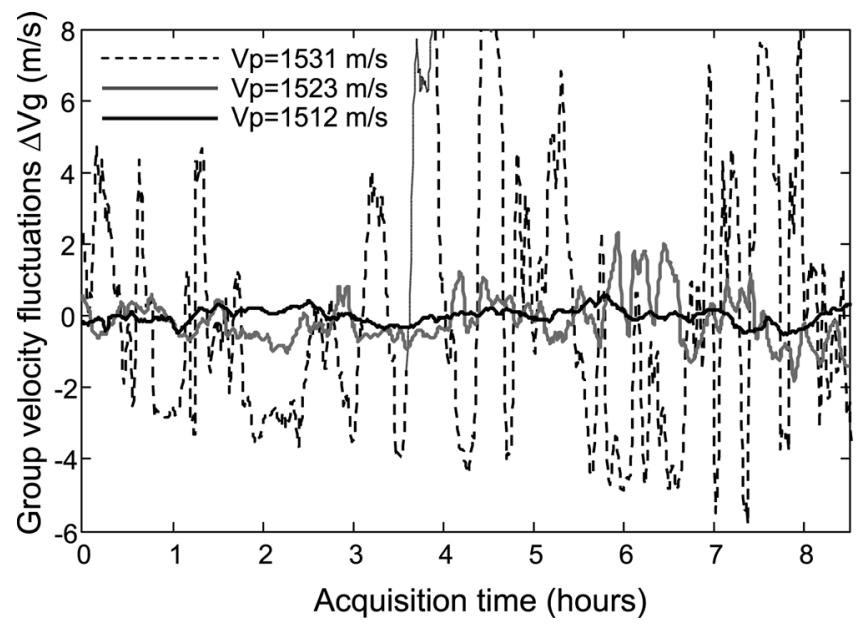

FIG. 8. Time evolution of the group velocity fluctuations $\Delta \mathrm{Vg}$ along the $8.5 \mathrm{~h}$ of acoustic acquisition for the three different phase velocities $\mathrm{Vp}$, as indicated.

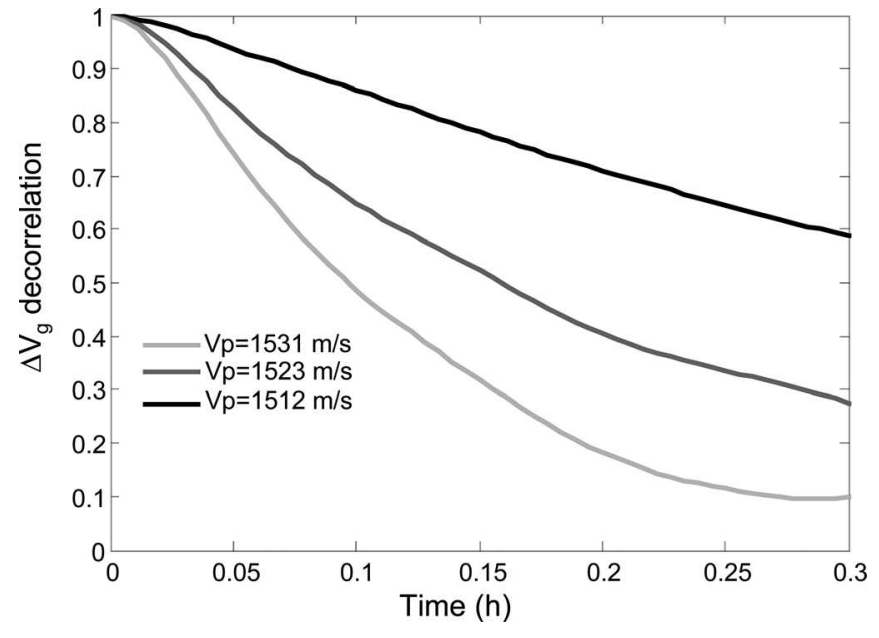

FIG. 9. Correlation function averaged over the $8.5 \mathrm{~h}$ of acoustic acquisition of the group velocity fluctuations $\Delta \mathrm{Vg}$ for the three different phase velocities $\mathrm{Vp}$, as indicated.

sitivity kernel (TSK) for the relevant eigenray paths. The TSK represents the travel-time change between a source and a receiver associated with a local sound speed change in the water column (Skarsoulis and Cornuelle, 2004). Ray theory itself does not include any diffraction effects adjacent to rays. However the spatial shape of the diffraction-based sensitivity kernel indicates the Fresnel zone that must be taken into account for the estimation of acoustic fluctuations in a specific ocean environment.

In recent years, several papers suggested that higherresolution tomography images can be obtained from this improved description of the forward model at a higher computational cost (Iturbe et al., 2009a; Marandet et al., 2011; Sarkar et al., 2012).

Since the TSK formulation was recently published (see, for example, Roux et al., 2011) and goes beyond the scope of the present discussion, this study was limited to the computation of the TSK for two eigenrays with turning points at thermoclines $\mathrm{A}$ and $\mathrm{B}$, respectively. In practice, the TSKs were calculated from a parabolic equation (PE) computation in the shallow-water environment shown in Fig. 1(a) and with the average sound speed profile in Fig. 1(b). The PE computation was performed between two source-receiver arrays centered at a depth of $90 \mathrm{~m}$ with an array length similar to the subarray length used for the DBF analysis with the FAF05 data.

As expected, the TSK in Fig. 11 shows that the sensitivity to sound speed fluctuations is mostly located around the eigenray path with a Fresnel zone size that depends on the central frequency, the bandwidth, and the size of the sourcereceiver arrays (Iturbe et al., 2009b). For the first beam [Fig. 11(a)], the travel-time sensitivity is strongly concentrated at the turning point at a depth of $35 \mathrm{~m}$. For the second one [Fig. 11(b)], the travel-time sensitivity is spread over a greater area from a depth of 35 to $15 \mathrm{~m}$, between the two thermoclines. Although the maximum travel-time perturbation for the first beam is locally higher than the second [as seen from the color bar scales in Figs. 11(a) and 11(b)], the integrated travel-time perturbation in the region of the internal wave variability is 3 times higher for the second beam. In other words, with most of the internal wave fluctuations 


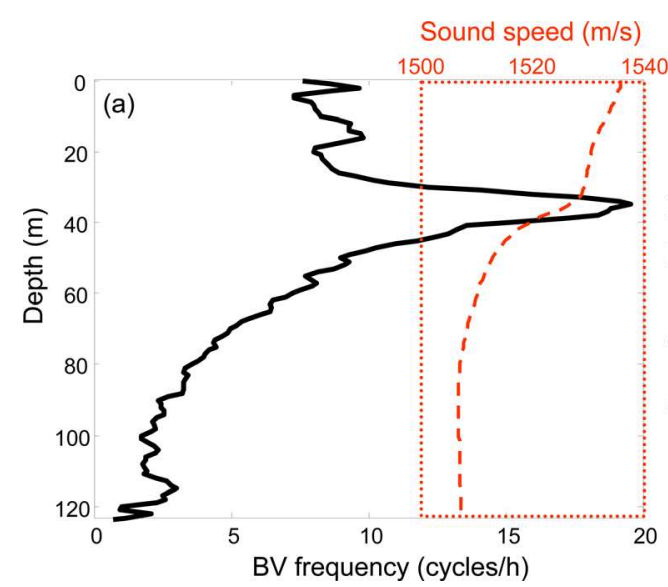

that occur between 15 and $35 \mathrm{~m}$, the beams crossing this water layer, instead of turning at its edge, accumulate more travel-time perturbations.

To summarize, it is the spatial spread of the TSK for the beam with turning point at thermocline $\mathrm{B}$ that explains the shorter time coherence observed with the acoustic data.
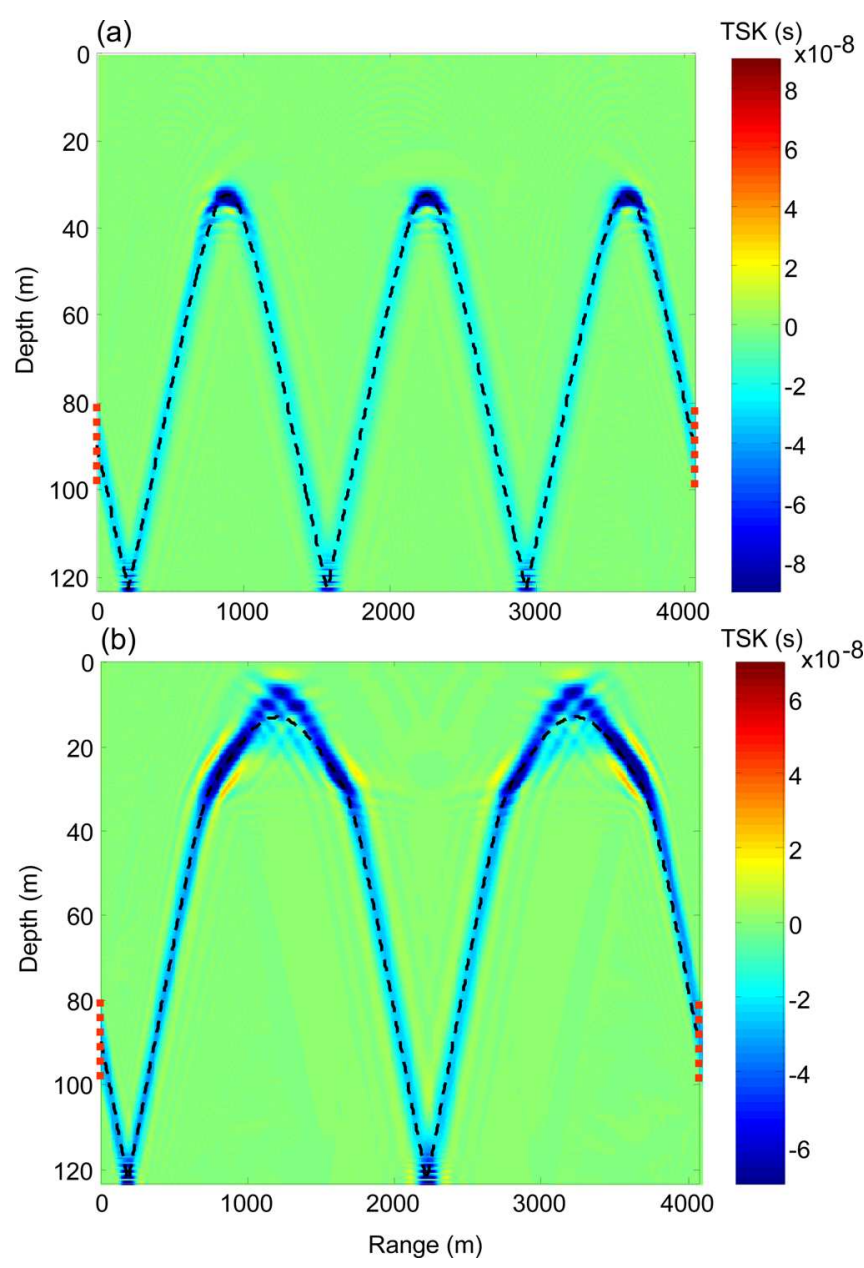

FIG. 11. (Color online) TSK computed for 2 eigenrays (dashed lines) with DBF performed from $20-\mathrm{m}$ long source-receiver arrays centered at depth $=90 \mathrm{~m}$ (see vertical dots at range $=0$ and range $=4071 \mathrm{~m}$ ). From the ray path computation, we have: (a) $\mathrm{Vp}=1526 \mathrm{~m} / \mathrm{s}$ and $\mathrm{Vg}=1497 \mathrm{~m} / \mathrm{s}$; (b) $\mathrm{Vp}=1533 \mathrm{~m} / \mathrm{s}$ and $\mathrm{Vg}=1506 \mathrm{~m} / \mathrm{s}$, with turning points at thermocline A and B, respectively [see Fig. 1(b)]. The TSKs are calculated at $3 \mathrm{kHz}$ for a $50 \%$ bandwidth. The color bar represents the travel-time change in seconds for a local sound speed increase of $1 \mathrm{~m} / \mathrm{s}$ of a volume of $1 \mathrm{~m}^{3}$ of water.

\section{CONCLUSIONS}

Through array beamforming that is simultaneously performed on a SA and a RA in a shallow-water fluctuating environment, a set of eigenrays were isolated that characterize the bulk of the acoustic fluctuations. The combination of acoustic data analysis and the characterization of the oceanic environment shows that the dominant source of fluctuations is related to the BV profile and internal wave variability, as seen by their effects on the eigenray travel-times.

The travel-time variability of the $\mathrm{Vg}$-Vp fluctuations are qualitatively consistent with the internal wave variability, including the maximum frequency allowed by the BV profile observed by the CTD casts. A more quantitative analysis would involve the use of many beams to estimate the structure of the internal wave variability in the dataset but the analysis was limited here to a consistency check. The structure of the TSK explains the otherwise confusing result that beams turning above the strongest thermocline have more variability and a shorter decorrelation time.

Furthermore, the eigenrays that fluctuate the most are connected with a particular region of the Vg-Vp curve. This region corresponds to a transition between refracted and reflected eigenrays that is representative of a major change in the waveguide invariant $(\beta)$. Because $\beta$ is known to be robust, the use of such $\mathrm{Vg}-\mathrm{Vp}$ analysis to infer acoustic fluctuations associated with internal wave propagation should be true for any shallow water environment.

\section{ACKNOWLEDGMENTS}

This project was supported by the Office of Naval Research (ONR). This study was performed in collaborative experiments with the NATO Underwater Research Centre (NURC), La Spezia, Italy, with Mark Stevenson as Chief Scientist. Scientists who contributed to these experiments include Tuncay Akal, Piero Boni, Piero Guerrini, other NURC staff, and the officers and crew of the RV Alliance.

Aulanier, F., Nicolas, B., Roux, P., and Mars, J. I. (2011). "Perspective of tomography inversion using direction-of-arrival and direction-ofdeparture,” J. Acoust. Soc. Am. 130, 2392.

Badiey, M., Katsnelson, B. G., Lynch, J. F., and Perselkov, S. (2007). "Frequency dependence and intensity fluctuations due to shallow water internal waves," J. Acoust. Soc. Am. 122, 747-760. 
Brekhovskikh L. M., and Lysanov, Y. P. (2003). Fundamentals of Ocean Acoustics, 3rd ed. (Springer-Verlag, New York), Chaps. 5 and 6, pp. 105$112 ; 136-148$

Chuprov S. D. (1982). "Interference structure of a sound field in a layered ocean," in Ocean Acoustics, Current State, edited by L. M. Brekhovskikh and I. B. Andreevoi (Nauka, Moscow), pp. 71-91.

Cockrell, K. L., and Schmidt, H. (2010). "Robust passive range estimation using the waveguide invariant," J. Acoust. Soc. Am. 127, 2780-2789.

D’Spain, G. L., and Kuperman, W. A. (1999). "Application of waveguide invariants to analysis of spectrograms from shallow water environments that vary in range and azimuth," J. Acoust. Soc. Am. 106, 2454-2468.

Fupo, W., and Yue, P. (2011). "Real time target localization using waveguide invariant in shallow water," in IEEE International Conference on Signal Processing, Communications and Computing, pp. 1-4.

Grachev G. A. (1993). "Theory of acoustic field invariants in layered waveguides," Acoust. Phys. 39, 33-35.

Hailiang, T., and Krolik, J. (2007). "Waveguide invariant focusing for broadband beamforming in an oceanic waveguide," J. Acoust. Soc. Am. 123, 1338-1346.

Harrison C. H. (2011). "The relation between the waveguide invariant, multipath impulse response, and ray cycles," J. Acoust. Soc. Am. 129, 2863-2877.

Hodgkiss, W. S., Ensberg, D. E., D’Spain, G. L., Booth, N. O., and Schey, P. W. (1995). "Comparison of acoustic and non-acoustic methods of vertical line array element localization," in Proceedings of Oceans' 95, San Diego, CA, pp. 1296-1302.

Iturbe, I., Roux, P., Nicolas, B., Virieux, J., and Mars, J. I. (2009a). "Shallow-water acoustic tomography performed from a doublebeamforming algorithm,” IEEE J. Ocean. Eng. 34, 140-149.

Iturbe, I., Roux, P., Virieux, J., and Nicolas, B. (2009b). "Travel-time sensitivity kernels vs diffraction patterns obtained through double beamforming in shallow water," J. Acoust. Soc. Am. 126, 713-720.

Jensen, F. B., Kuperman, W. A., Porter, M. B., and Schmidt, H. (2011). Computational Ocean Acoustics, 2nd ed. (Springer Science + Business Media, New York), Chaps. 2 and 5, pp. 133-138, 337-349, and 441.

Kim, S., Kuperman, W. A., Hodgkiss, W. S., Song, H. C., and Edelmann, G. F. (2003). "Robust time reversal focusing in the ocean," J. Acoust. Soc. Am. 114, 145-157.

Kuperman, W. A., D’Spain, G. L., and Heaney, K. D. (2001). "Long-range source localization from single hydrophone spectograms," J. Acoust. Soc. Am. 109, 1935-1943.

Kuperman, W. A., Kim, S., Edelmann, G. F., Hodgkiss, W. S., Song, H. C., and Akal, T. (2002). "Group and phase speed analysis for predicting and mitigating the effects of fluctuations," in Impact of Littoral Environment Variability on Acoustic Predictions and Sonar Performance, edited by J. Pace (Springer, New York), pp. 279-286.
Marandet, C., Roux, P., Nicolas, B., and Mars, J. I. (2011). "Target detection and localization in shallow water: An experimental demonstration of the acoustic barrier problem at the laboratory scale," J. Acoust. Soc. Am. 129, 85-97.

Quijano, J. E., Campbell, R. L., Oesterlein, T. G., and Zurk, L. M. (2010). "Experimental observations of active invariance striations in a tank environment," J. Acoust. Soc. Am. 128, 611-618.

Rouseff, D., and Leigh, C. V. (2002). "Using the waveguide invariant to analyze lofargrams," in Proceedings of the Oceans'02, Biloxi, MS, pp. IV. 2239-IV. 2243.

Rouseff, D., and Spindel, R. C. (2002). "Modeling the waveguide invariant as a distribution," in Ocean Acoustic Interference Phenomena and Signal Processing, edited by W. A. Kuperman and G. L. D'Spain (AIP, New York), Chap. 5, pp. 137-148.

Roussef, D., and Zurk, L. M. (2011). "Striation-based beamforming for estimating the waveguide invariant with passive sonar," J. Acoust. Soc. Am. 130, EL76-EL81.

Roux, P., Cornuelle, B. D., Kuperman, W. A., and Hodgkiss, W. S. (2008). "The structure of ray-like arrivals in a shallow-water waveguide," J. Acoust. Soc. Am. 124, 3430-3439.

Roux, P., Culver, R. L., and Walker, S. (2010). "Application of the Coherent-to-incoherent Intensity Ratio (CTIR) to estimating ocean surface roughness from high frequency, shallow water propagation measurements," J. Acoust. Soc. Am. 127, 1258-1266.

Roux, P., Iturbe, I., Nicolas, B., Virieux, J., and Mars, J. I. (2011). "Traveltime tomography in shallow water: Experimental demonstration at an ultrasonic scale," J. Acoust. Soc. Am. 130, 1232-1241.

Roux, P., Kuperman, W. A., Hodgkiss, W. S., Song, H. C., Akal, T., and Stevenson, M. (2004). "A non-reciprocal implementation of time reversal in the ocean," J. Acoust. Soc. Am. 116, 1009-1015.

Sarkar, J., Marandet, C., Roux, P., Walker, S., Cornuelle, B. D., and Kuperman, W. A. (2012). "Sensitivity kernel for surface scattering in a waveguide," J. Acoust. Soc. Am. 131, 111-118.

Shang, E. C., Wu, J. R., and Zhao Z. D. (2012). "Relating waveguide invariant and bottom reflection phase-shift parameter P in a Pekeris waveguide," J. Acoust. Soc. Am. 131, 3691-3697.

Skarsoulis, E., and Cornuelle, B. D. (2004). "Travel-time sensitivity kernels in ocean acoustic tomography," J. Acoust. Soc. Am. 116, 227-238.

Song, H. C., Kuperman, W. A., and Hodgkiss, W. S. (1998). "A time-reversal mirror with variable range focusing," J. Acoust. Soc. Am. 103, 3234-3240.

Thode, A. (2000). "Source ranging with minimal environmental information using virtual receiver and waveguide invariant theory," J. Acoust. Soc. Am. 108, 1582-1594.

Yang, T. C. (2003). "Beam intensity striations and applications," J. Acoust. Soc. Am. 113, 1342-1352. 